Article

\title{
The Feasibility of Contrast-to-Noise Ratio on Measurements to Evaluate CT Image Quality in Terms of Low-Contrast Detailed Detectability
}

\author{
Haney A Alsleem ${ }^{1}$ and Hussain M Almohiy ${ }^{2, *}$ \\ 1 Departments of Radiological Science, Colleges of Applied Medical Sciences, \\ Imam Abdulrahman Bin Faisal University, Dammam 34212, Saudi Arabia; hsleem@iau.edu.sa \\ 2 Departments of Radiological Science, Colleges of Applied Medical Sciences, King Khalid University, \\ Abha 62529, Saudi Arabia \\ * Correspondence: hmohiy@kku.edu.sa
}

Received: 27 March 2020; Accepted: 29 June 2020; Published: 6 July 2020

\begin{abstract}
Background: To evaluate contrast-to-noise ratio (CNR) measurements in assessing image quality, in the context of the detectability performance of low-contrast detail (LCD), in computed tomography (CT) images, since exposure to elevated ionising-type radiation is considered to present excessive carcinogenic risk, whilst also causing distress in study subjects. Methods: An LCD phantom module (CTP515) was utilised in the study. Three dissimilar contrast items were used to analyse the ramifications of the proportions of an object on the CNR. Three multidetector CT (MDCT) scanners were used, with 16-MDCT, 64-MDCT and 80-MDCT frameworks, respectively. The CT scans were recreated using three dissimilar remaking algorithms—soft, standard and lung. The effects exerted on the CNR by various remodelling algorithms, as well as the contrast of various objects along with the size of the objects, were explored. The Hounsfield units of each chosen object (one unit representing the outer portion of the object) and the background and the standard deviation of the noise parameter were quantified, and algorithms were developed using MATLAB. Results: The CNR information was greatly influenced by changing the image recreation calculations and was very much increased in the soft-tissue recreation images using 16-MDCT and 64-MDCT. The CNR information was also increased more in the optimum recreation images than in the reproduced images from the computational procedure used in the 80-MDCT. The results did not show any remarkable contrasts in the CNR values between the different object sizes. Overall, a higher $\mathrm{kVp}$ produced an improved CNR in all the CT scanners. In particular, there were prominent upgrades in the CNR information when the $\mathrm{kVp}$ was increased from 80 to 120 . Higher mAs levels gave better CNR values overall, especially for greater section thicknesses. Based on the CNR estimations, the 64-MDCT provided the best correlation among the CT scanners. Conclusions: The objective LCD appraisal method, based on CNR measurements, was confirmed as being useful for checking the different impacts of $\mathrm{kVp}, \mathrm{mAs}$ and section thickness on the nature of the picture. This procedure was similarly viable in assessing the impacts of the different reconstruction calculations and the different differentiation questions on the nature of the image.
\end{abstract}

Keywords: radiation dose; contrast-to-noise ratio (CNR); low-contrast detail (LCD); multidetector computed tomography (MDCT)

\section{Introduction}

A significant increase in the number of computed tomography (CT) scanners has highlighted the need to reduce the amount of radiation used in them [1]. While the evolution of the CT technique has resulted in the enhanced diagnosis of disease, its disadvantage has been the corresponding radiation 
dosage [2]. Numerous analysts have suggested rational scan protocols for regular CT investigations and expressed a desire for the various manufacturers to guarantee high image quality using less radiation $[3,4]$. Consequently, radiation doses can now be lowered without sacrificing diagnostic details in the image [3]. One solution for improving image quality at low-radiation levels has relied on progress being made in the rebuilding methods [5-8], among which iterative reconstruction (IR) algorithms are commonly used. In addition, several studies have recommended that low-contrast detail (LCD) detection capability might be the most suitable method for standardising images [9-11]. LCD refers to the capability of a CT scan to differentiate between various objects that have similar X-ray attenuation coefficients. The most serious and noteworthy challenge in this aspect is that techniques utilising low dose decline the detectible performance of LCD, uniquely in abdomen examinations. For example, carcinogenic disease of the liver is customarily demonstrated as low-attenuation lesions inside a background of slightly higher attenuation normal tissue. Detectible capacity of LCD must be preserved with any dose-reduction strategy. Contrast-to-noise ratio (CNR) is an important tool used to determine image quality. It is a measure of image quality established on a contrast. Prior studies have shown that images obtained using hybrid-type IR (HIR) algorithms, which comprise the majority of various accessible IR procedures, are able to maintain image standards using a decrease in radiation of $20-25 \%$ to $65-67 \%$, although some image noise and artefacts remain $[12,13]$. Recently, iterative-type model reproduction (IMR), an information-based IR calculation, has enabled an additional decrease in radiation dosage with enhanced image representation, although this has resulted in a notable decrease in contrast and a suppression of artefacts that are differentiated into filtered back projection in the period.

Filtered back projection (FBP) and HIR calculations in LCD scans [14-18]. The two foremost methods—subjective and objective-are accessible for computing the LCD of various CT images [19]. The subjective technique depends on human perception, and the objective one is based on quantitative measurements of contrast-to-noise ratio (CNR) [9]. Because of the subjectivity of human observers, the objective way is considered to be more suitable for dealing with estimating LCD execution. In this study, we aimed to assess the impacts of the settings-essentially, milliampere-second (mAs) and Kilovoltage peak $(\mathrm{kVp})$ - on the LCD of CT scanners. The strategy used was based on the CNR estimations of scanned objects. We also examined the differences between different CT scanners, in terms of the CNR estimations of those scanned objects. Our overarching aim was thus to evaluate the methodology used in CNR measurements in order to assess image quality in terms of the detectability of LCD in CT images.

\section{Materials and Methodology}

\subsection{The Phantom Model}

The Catphan ${ }^{\circledR} 600$ (Phantom Laboratory, Greenwich, NY, USA)—known as the phantom—was mainly used in this study (Figure 1). In this, the image is developed from a medium of solid cast, produced using modules with a diameter of $15 \mathrm{~mm}$. Each module assesses particular audits that correlate with the usage capability of the multidetector CT (MDCT). An LCD image module (CTP-515) was installed in the phantom for use in the experiment (as shown in Figure 1). The image shows a collection of sets of round, empty, low, multifaceted natural objects, arranged on two levels in the phantom. The objects were 40-mm high, were separated by different spaces (i.e., 2-9 and $15 \mathrm{~mm}$ ) and were examined at three contrast levels $(0.3 \%, 0.5 \%$ and $1 \%)$. The objects arranged around the outer level were chosen for study, those measuring $5-15 \mathrm{~mm}$ at a $1 \%$ contrast level, those $6-15 \mathrm{~mm}$ at a $0.5 \%$ level and those $7-15 \mathrm{~mm}$ at $0.3 \%$. Thus, three specific contrast levels were used to examine the objects to assess the effect of object size on the CNR. The objects chosen for this part of the study were $1 \%$ separate level things for two purposes. 


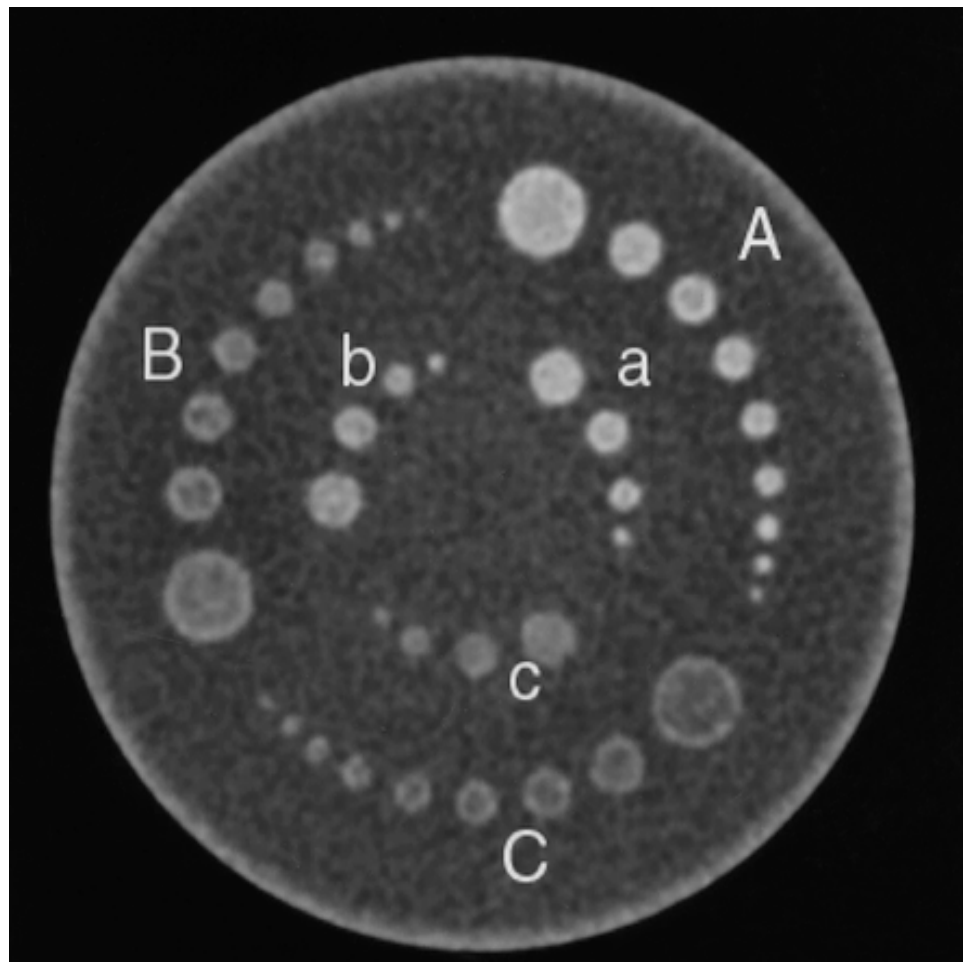

Figure 1. A Phantom of low contrast module. Six different regions have been depicted where low contrast objects are put (A-C and $\mathrm{a}-\mathrm{c}$ ). Low cylindrical objects were placed in Regions $\mathrm{A}-\mathrm{C}$ to contrast the difference in the objects in Regions $\mathrm{A} / \mathrm{a}, \mathrm{B} / \mathrm{b}$ and $\mathrm{C} / \mathrm{c}$ with the backgrounds of $1 \%, 0.5 \%$ and $0.3 \%$, respectively.

\subsection{The CT Scanners}

Three MDCT scanners-a 16-MDCT structure (GE Lightspeed VCT; GE Healthcare, Milwaukee, WI, USA) a 64-MDCT structure (Light Speed VCT, GE Healthcare) and an 80-MDCT structure (Aquilion-80, Toshiba, American Medical Systems Inc., Minnetonka, MN, USA)—were used (as shown in Table 1). All systems were well maintained. This ensured that the scanners' capabilities met the manufacturers' specifications.

Table 1. Specifications of the CT Scanner.

\begin{tabular}{cccc}
\hline & 16-MDCT & 64-MDCT & 80-MDCT \\
\hline Manufacturer's name & GE Healthcare & GE Healthcare & Toshiba \\
\hline Name of the product & LightSpeed 16 & LightSpeed VCT 64 & Aquilion Prime 80 \\
\hline Type of Detector & $\begin{array}{c}\text { Solid-state polycrystalline } \\
\text { ceramic scintillator }\end{array}$ & Solid-state ceramic & Solid-state Gd2O2S \\
\hline Cell size of the Detector & $0.625 \mathrm{~mm}$ & $0.625 \mathrm{~mm}$ & $0.5 \mathrm{~mm}$ \\
\hline Coverage area & $20 \mathrm{~mm}$ & $40 \mathrm{~mm}$ & $40 \mathrm{~mm}$ \\
\hline Aperture of the Gantry & $70 \mathrm{~cm}$ & $70 \mathrm{~cm}$ & $78 \mathrm{~cm}$ \\
\hline Algorithm & Filtered back projection, 2D back & GE property volume & Filtered back projection \\
reconstruction & $\begin{array}{c}0.7 \times 0.6 \\
0.9 \times 0.7\end{array}$ & $\begin{array}{c}0.6 \times 0.7 \\
0.9 \times 0.9\end{array}$ & $\begin{array}{c}0.9 \times 0.8 \\
1.6 \times 1.4\end{array}$ \\
\hline Focal spot size & & &
\end{tabular}

\subsection{Image Acquisition}

The unit of the LCD belonging to the particular phantom was assembled inside the scanner portion of the gantry. The complete measurements were made utilising two voltage increments of 
80 and $120 \mathrm{kVp}$ at various levels of $\mathrm{mAs}$ and different section thicknesses as well as reconstruction computations (as depicted in Table 2). The field of view was set at $360 \mathrm{~mm}$ for the 16-MDCT and 64-MDCT scanners and at $240 \mathrm{~mm}$ for the 80-MDCT scanner for data collection. The data were then matched up, using three specific changing configurations-standard being the initial one, soft tissue being the second and lung being the last. The effects on the CNR of the various multiple counts and separate objects, together with object size, were examined, as was the impact of $\mathrm{kVp}, \mathrm{mAs}$ and section thickness.

Table 2. The Protocol parameters utilised for image acquisition.

\begin{tabular}{ccc}
\hline Voltage kVp & \multicolumn{2}{c}{80 and 120} \\
\hline mAs Utilised & \multicolumn{3}{c}{ 10, 20, 50, 100 and 200 } \\
\hline \multirow{2}{*}{ Section thickness } & For the 80-MDCT (in mm) & $0.5,1,2$ and 5 \\
\cline { 2 - 3 } & For 16-MDCT and the 64-MDCT (in mm) & $0.625,1.25,2.5$ and 5 \\
\hline Reconstruction algorithms used & \multicolumn{2}{c}{ Standard, lung and soft tissue } \\
\hline
\end{tabular}

\subsection{Calculation of the CNR and MATLAB Software}

The universal Hounsfield units (HU) of each chosen object (i.e., the outer-level articles), the background and the standard deviation (SD) component (i.e., noise) were evaluated. The required calculations to process the CNR data were made using MATLAB (v.7.14, MathWorks, Natick, MA, USA). The calculations were then applied to each image, and the CNR for everything was resolved using the equation [20] below. The SD of the mean CNR was also computed.

$$
\mathrm{CNR}=\frac{\mathrm{CT} \text { value }(\text { object })-\mathrm{CT} \text { value }(\text { background })}{\mathrm{SD}(\text { noise })}
$$

\subsection{Statistics and Analysis}

The Gaussian statistical distribution formula was used to determine the dispersion ordinariness of the arithmetic for each factor. Gaussian dispersion examines the likelihood of whether the arithmetic for each factor remains between two genuine cut-off points [21]. A two-route between-bunches examination of difference (using SPSS programming) enabled an interrogation of the data. An analysis of the variance (ANOVA) of the two-way-measurement analysis was performed to look at the effects of the various CT convention parameters, including reproduction of the calculations, the $\mathrm{mAs}, \mathrm{kVp}$ and section thickness, on the CNR measurements of each image and each object in the images. The effects of different factors, such as object size, object level and type of scanner, were also examined by two-way ANOVA. The two-way ANOVA was utilised to calculate whether notable contrasts subsisted between $\mathrm{kVp}$ bunches revealed between similar types of $\mathrm{mAs}$ and section thicknesses, between interconnecting $\mathrm{mAs}$ bunches at equivalent $\mathrm{kVp}$ and section thicknesses and between section thickness bunches at analogous $\mathrm{mAs}$ and $\mathrm{kVp}$. A similar type of analysis was also used to investigate whether there was a critical correlative impact between these factors. A $t$-test, at an alpha estimation of approximately 0.05 , was used as part of the two-way ANOVA computations to determine whether centrality contrasts existed between the various groupings [21].

\section{Results}

The effects of the size of the objects, with $1 \%$ contrast level, on CNR values were evaluated for different MDCT scanners, reconstruction algorithms, object contrast levels and $\mathrm{mAs}$ selections (Figure 2 and Tables 3 and 4). Changes in the CNR values for 16-MDCT with 8-mm object size and for 80-MDCT with 5-mm object size can be seen in Figure 2. Data from the soft-tissue and reproducing-algorithm images and objects at $1 \%$ contrast are recorded in Tables 3 and 4. 


\section{CNR of $1 \%$ contrast level objects of different size in different MDCT scanners}

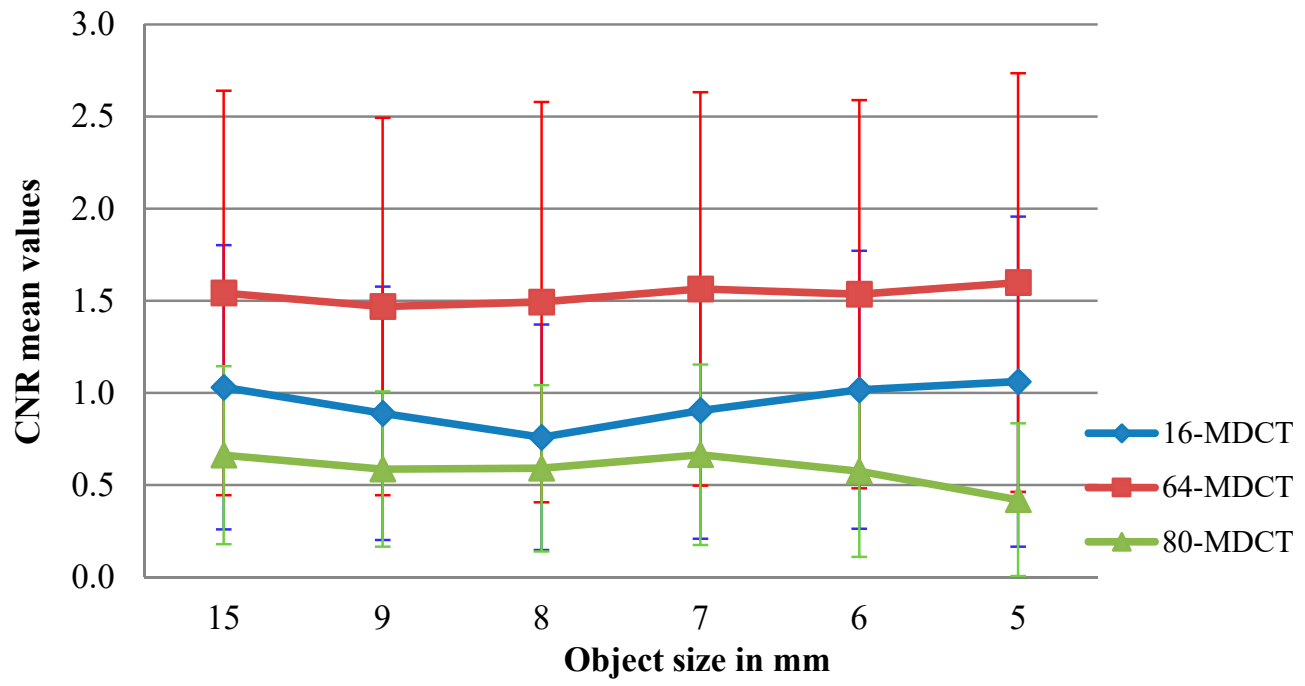

Figure 2. Limited effects of the size of $1 \%$ contrast levels objects on CNR values for all CT scanners. (Note the change in CNR values for 16-MDCT with 8-mm object size and for 80-MDCT with 5-mm object size.)

Table 3. Mean CNR values of the images at $80 \mathrm{kVp}$. The mean values were obtained by averaging three identical exposures.

\begin{tabular}{ccccccccccc}
\hline & & \multicolumn{3}{c}{ 16-MDCT } & \multicolumn{3}{c}{ 64-MDCT } & \multicolumn{2}{c}{ 80-MDCT } \\
\cline { 3 - 11 } kVp & mAs & $\begin{array}{c}\text { Section } \\
\text { Thickness }\end{array}$ & Mean & SD & $\begin{array}{c}\text { Section } \\
\text { Thickness }\end{array}$ & Mean & SD & $\begin{array}{c}\text { Section } \\
\text { Thickness }\end{array}$ & Mean & SD \\
\hline 80 & 10 & 0.625 & 0.24 & 0.18 & 0.625 & 0.26 & 0.29 & 0.5 & -0.02 & 0.20 \\
80 & 10 & 5 & 0.18 & 0.24 & 5 & 0.56 & 0.22 & 5 & 0.19 & 0.17 \\
80 & 20 & 0.625 & 0.07 & 0.21 & 0.625 & 0.52 & 0.43 & 0.5 & 0.11 & 0.25 \\
80 & 20 & 5 & 0.50 & 0.21 & 5 & 0.89 & 0.23 & 5 & 0.52 & 0.19 \\
80 & 50 & 0.625 & 0.32 & 0.38 & 0.625 & 0.61 & 0.39 & 0.5 & 0.27 & 0.21 \\
80 & 50 & 5 & 0.94 & 0.16 & 5 & 1.53 & 0.23 & 5 & 0.72 & 0.15 \\
80 & 100 & 0.625 & 0.74 & 0.30 & 0.625 & 1.04 & 0.36 & 0.5 & 0.29 & 0.15 \\
80 & 100 & 5 & 1.31 & 0.19 & 5 & 2.19 & 0.27 & 5 & 0.91 & 0.17 \\
80 & 200 & 0.625 & 0.83 & 0.34 & 0.625 & 1.20 & 0.25 & 0.5 & 0.53 & 0.14 \\
80 & 200 & 5 & 1.96 & 0.28 & 5 & 3.11 & 0.39 & 5 & 1.42 & 0.17 \\
\hline
\end{tabular}

Table 4. Mean CNR values of the images at $120 \mathrm{kVp}$. The mean values were obtained by averaging three identical exposures.

\begin{tabular}{ccccccccccc}
\hline & & \multicolumn{3}{c}{ 16-MDCT } & \multicolumn{3}{c}{ 64-MDCT } & \multicolumn{2}{c}{ 80-MDCT } \\
\cline { 3 - 10 } kVp & mAs & $\begin{array}{c}\text { Section } \\
\text { Thickness }\end{array}$ & Mean & SD & $\begin{array}{c}\text { Section } \\
\text { Thickness }\end{array}$ & Mean & SD & $\begin{array}{c}\text { Section } \\
\text { Thickness }\end{array}$ & Mean & SD \\
\hline 120 & 10 & 0.625 & 0.35 & 0.36 & 0.625 & 0.57 & 0.30 & 0.5 & 0.06 & 0.21 \\
120 & 10 & 5 & 0.58 & 0.24 & 5 & 1.14 & 0.34 & 5 & 0.35 & 0.20 \\
120 & 20 & 0.625 & 0.49 & 0.36 & 0.625 & 0.71 & 0.38 & 0.5 & 0.23 & 0.16 \\
120 & 20 & 5 & 1.02 & 0.21 & 5 & 1.60 & 0.32 & 5 & 0.65 & 0.26 \\
120 & 50 & 0.625 & & 0.46 & 0.625 & 1.09 & 0.43 & 0.5 & 0.37 & 0.19 \\
120 & 50 & 5 & 1.56 & 0.22 & 5 & 2.54 & 0.22 & 5 & 1.05 & 0.23 \\
120 & 100 & 0.625 & 0.98 & 0.31 & 0.625 & 1.39 & 0.32 & 0.5 & 0.64 & 0.24 \\
120 & 100 & 5 & 2.51 & 0.25 & 5 & 3.48 & 0.27 & 5 & 1.33 & 0.31 \\
120 & 200 & 0.625 & 1.27 & 0.29 & 0.625 & 2.17 & 0.47 & 0.5 & 0.80 & 0.28 \\
120 & 200 & 5 & 3.17 & 0.43 & 5 & 5.14 & 0.33 & 5 & 1.87 & 0.40 \\
\hline
\end{tabular}


The effects of object size on the CNR values were examined at first. Object size in relation to different scanners, reconstruction calculations, levels of object contrast and mAs was also scrutinised. The effect of the reconstruction calculations, $\mathrm{mAs}, \mathrm{kVp}$ and section thickness on the CNR data were then assessed. The output of the various scanners was then compared, based on the CNR values.

\subsection{Effect of Object Size on CNR Values}

The effect of object size on the CNR values, at a 1\% contrast level, was assessed for each MDCT scanner, for the various reconstruction calculations, contrast levels on the objects and mAs values. It was found that negligible variations in the mean CNR values occurred for the objects of various sizes across all scanners ( $p$ value $>0.1$ ) (see Table 5$)$, but there were considerable variations ( $p$ value $=0.021$ ) between the 5 - and $8-\mathrm{mm}$ objects at $1 \%$ contrast in the 16-MDCT. In the 64-MDCT, there was always a negligible difference in CNR values among the objects of different dimensions. In the 80-MDCT, there were significant contrasts in the CNR values between a 5-mm object and the 8- and 15-mm objects.

Table 5. Differences in CNR values ( $p$ values, student $t$-tests) between different object sizes at $1 \%$ contrast in each CT scanner.

\begin{tabular}{ccccc}
\hline \multirow{2}{*}{ Object Size } & Object Sizes & \multicolumn{3}{c}{ Sig. ( $p$ Values, Student $t$-Tests) } \\
\cline { 3 - 5 } & & 16-MDCT & 64-MDCT & 80-MDCT \\
\hline \multirow{2}{*}{5} & 6 & 0.989 & 0.99 & 0.005 \\
& 7 & 0.228 & 0.999 & 0 \\
& 8 & 0 & 0.905 & 0.001 \\
& 9 & 0.145 & 0.799 & 0.002 \\
& 15 & 0.998 & 0.994 & 0 \\
\hline \multirow{2}{*}{6} & 7 & 0.601 & 1 & 0.309 \\
& 8 & 0.004 & 0.998 & 0.999 \\
& 9 & 0.459 & 0.987 & 1 \\
7 & 15 & 1 & 1 & 0.333 \\
\hline \multirow{2}{*}{7} & 8 & 0.308 & 0.982 & 0.537 \\
& 9 & 1 & 0.937 & 0.467 \\
& 15 & 0.472 & 1 & 1 \\
\hline 9 & 9 & 0.436 & 1 & 1 \\
\hline \multirow{2}{*}{9} & 15 & 0.002 & 0.997 & 0.566 \\
\hline
\end{tabular}

\subsection{Impact of Image Reconstruction Calculations on CNR Values}

In the 64-MDCT and corresponding 16-MDCT, the CNR values for the reconstructed soft-tissue images were substantially higher than those for the corresponding lung images ( $p$ value $<0.001$ ). The soft-tissue images had impressively higher CNR values than the standard images in the 16-MDCT and 64-MDCT ( $p$ values $=0.011$ and $<0.001$, respectively). In the 80-MDCT, however, the standard images had much higher CNR estimates than the other types of images ( $p$ value $<0.001)$.

\subsection{Impact of $k V p$ on $C N R$ Values}

Using a higher $\mathrm{kVp}$ gave higher CNR values in all CT scanners. There were critical increases in the CNR values when the $\mathrm{kVp}$ was increased from 80 to 120 (Table 6). On the other hand, there was little separation between the CNR values at levels of 80 and $120 \mathrm{kVp}$ at $10 \mathrm{mAs}$ and corresponding $0.625-\mathrm{mm}$ section thickness ( $p$ value $=1$ ) within the 16-MDCT. Considering $100 \mathrm{mAs}$ and corresponding $0.625 \mathrm{~mm}$, there were, however, inconsequential distinctions in the CNR values when the kVp was increased to 120 ( $p$ value $=0.894)$. Considering the 64-MDCT, wide variations in the CNR values were seen when the $\mathrm{kVp}$ was elevated from 80 to $120 \mathrm{kVp}$, as well as one significant occurrence at $20 \mathrm{mAs}$ and $0.625-\mathrm{mm}$ thickness ( $p$ value $<0.639$ ). In the 80-MDCT, there were insignificant increases in the 
CNR values at 10 and $20 \mathrm{mAs}$ for each section during $\mathrm{kVp}$ increment from 80 to $120 \mathrm{kVp}$. Considering $50 \mathrm{mAs}$ and 0.5 - and 1-mm section thicknesses, there were inconsequential elevations occurring to the CNR values ( $p$ values $=0.51$ and 0.77 , respectively) for elevations from 80 to $120 \mathrm{kVp}$. In addition, further increments in the CNR values at 80 and $120 \mathrm{kVp}$ were seen at $100 \mathrm{mAs}$ and $0.5-\mathrm{mm}$ section thickness (the $p$ value $=0.81$ ).

Table 6. The dissimilarities (student $t$-tests, $p$ values) between images using the same quantity of mAs and section thicknesses with varying $\mathrm{kVp}$ in each $\mathrm{CT}$ scanner.

\begin{tabular}{ccccc}
\hline (J) Image Code & (I) Image Code & \multicolumn{2}{c}{ Sig. $(\boldsymbol{p}$ Values, Student $t$-Tests) } \\
\cline { 3 - 5 } (kVp-mAs-Section Thickness) & $\mathbf{( k V p - m A s - S e c t i o n ~ T h i c k n e s s ) ~}$ & 16-MDCT & 64-MDCT & 80-MDCT \\
\hline $80-10-0.625 / 0.5$ & $120-10-0.625 / 0.5$ & 1.000 & 0.050 & 0.923 \\
$80-10-5$ & $120-10-5$ & 0.031 & 0.000 & 0.254 \\
$80-20-0.625 / 0.5$ & $120-20-0.625 / 0.5$ & 0.011 & 0.639 & 0.603 \\
$80-20-5$ & $120-20-5$ & 0.000 & 0.000 & 0.508 \\
$80-50-0.625 / 0.5$ & $120-50-0.625 / 0.5$ & 0.000 & 0.000 & 0.769 \\
$80-50-5$ & $120-50-5$ & 0.000 & 0.000 & 0.000 \\
$80-100-0.625 / 0.5$ & $120-100-0.625 / 0.5$ & 0.894 & 0.024 & 0.000 \\
$80-100-5$ & $120-100-5$ & 0.000 & 0.000 & 0.000 \\
$80-200-0.625 / 0.5$ & $120-200-0.625 / 0.5$ & 0.005 & 0.000 & 0.018 \\
$80-200-1.25 / 1$ & $120-200-1.25 / 1$ & 0.002 & 0.000 & 0.000 \\
$80-200-2.5 / 2$ & $120-200-2.5 / 2$ & 0.000 & 0.000 & 0.000 \\
$80-200-5$ & $120-200-5$ & 0.000 & 0.000 & 0.000 \\
\hline
\end{tabular}

\subsection{Impact of $m A$ s on $C N R$ Values}

Increased mAs levels increased the CNR values, especially at higher section thicknesses, in all CT scanners. Several significant increases in CNR values occurred when the mAs was increased from 10 to $20 \mathrm{mAs}$, initially, and then to 50, 100 or $200 \mathrm{mAs}$ (Table 7). Ultimately, there was inconsequential variations in the values of CNR between 10 and $20 \mathrm{mAs}$, especially at the lowest $\mathrm{kVp}$ and with progressively more thinner thicknesses (the $p$ value $>0.1$ ) in all corresponding CT scanners. Considering the 16-MDCT, there were inconsequential variations at 10 and $50 \mathrm{mAs}$ as well as at $80 \mathrm{kVp}$, when using 0.625- and 1.25-mm thicknesses of the sections ( $p$ values $=0.914$ and 0.244 ). Considering $120 \mathrm{kVp}$, there were inconsequential changes in the values of CNR at 10 and $20 \mathrm{mAs}$ as well as with $0.625,1.25$ and $2.5 \mathrm{~mm}$ thicknesses (the $p$ values $=0.76,1$ and 0.6 , respectively). In the 64-MDCT, insignificant variations were present between images at 10 and $20 \mathrm{mAs}$ and $120 \mathrm{kVp}$, with a $0.625-\mathrm{mm}$ section thickness ( $p$ value $=0.781$ ). In the $80-\mathrm{MDCT}$, there were insignificant variations between images at 10 and $50 \mathrm{mAs}$ and $120 \mathrm{kVp}$, with a $1.25-\mathrm{mm}$ thickness ( $p$ value $=0.643$ ).

\subsection{Impact of Section Thickness on CNR Values}

Thicker sections produced increased CNR values in almost all the CT scanners. Several significant increases in CNR values occurred when the section thickness was increased from 0.625 to $1.25,2.5$ or $5 \mathrm{~mm}$ (Table 8). In the 16-MDCT, there was insignificant differentiation between the CNR values for the $0.625-$ and $1.25-\mathrm{mm}$ section thicknesses at 20,50 and $100 \mathrm{mAs}$ and $80 \mathrm{kVp}$, as well as at 10 , 20, 50 and $200 \mathrm{mAs}$ and $120 \mathrm{kVp}$. There were also inconsequential differences when comparing the images with 0.625- and 1.25-mm section thicknesses at 20,50, 100 and $200 \mathrm{mAs}$ and $80 \mathrm{kVp}$, as well as at 10,20, 50 and $200 \mathrm{mAs}$ and $120 \mathrm{kVp}$. Inconsequential peaks were seen between the images utilising 0.625 - and 2.5-mm section thicknesses at 10 and $50 \mathrm{mAs}$ and $80 \mathrm{kVp}$, as well as at 10, 20 and $50 \mathrm{mAs}$ and $120 \mathrm{kVp}$. In addition, there were inconsequential peaks between the images using $0.625-$ and 5-mm section thicknesses at $10 \mathrm{mAs}$ and 80 and $120 \mathrm{kVp}$ (Table 8). In the 64-MDCT, there were inconsequential variations in the CNR values in some places for the $0.625-$ and $1.25-\mathrm{mm}$ thicknesses and for the $0.625-$ and $2.5-\mathrm{mm}$ thicknesses at 10 and $20 \mathrm{mAs}$ and $80 \mathrm{kVp}$. There were similarly inconsequential distinctions between images for the $0.625-$ and $1.25-\mathrm{mm}$ thicknesses at $100 \mathrm{mAs}$ and 
$80 \mathrm{kVp}(p$ value $=0.138)$. There were also insignificant distinctions between the images of the 0.625and 1.25-mm section thicknesses at $10 \mathrm{mAs}$ and $120 \mathrm{kVp}$ (Table 8).

Table 7. Dissimilarities ( $p$ values, student $t$-tests) between images utilising the same $\mathrm{kVp}$ and $\mathrm{mAs}$ with $\mathrm{mAs}$ variations in each CT scanner.

\begin{tabular}{ccccc}
\hline \multirow{2}{*}{$\begin{array}{c}\text { Image Code } \\
\text { kVp-mAs-ST }\end{array}$} & Image Code & \multicolumn{3}{c}{ Sig. $(\boldsymbol{p}$ Values, Student $t$-Tests) } \\
\cline { 3 - 5 } kVp-mAs-ST & 16-MDCT & 64-MDCT & 80-MDCT \\
\hline $80-10-0.625 / 0.5$ & $80-20-0.625 / 0.5$ & 0.395 & 0.203 & 0.310 \\
& $80-200-0.625 / 0.5$ & 0.000 & 0.000 & 0.000 \\
$80-10-1.25 / 1$ & $80-20-1.25 / 1$ & 0.001 & 0.347 & 0.886 \\
& $80-200-1.25 / 1$ & 0.000 & 0.000 & 0.000 \\
$80-10-2.5 / 2$ & $80-20-2.5 / 2$ & 0.188 & 0.099 & 0.120 \\
& $80-200-2.5 / 2$ & 0.000 & 0.000 & 0.000 \\
$80-10-5$ & $80-20-5$ & 0.000 & 0.004 & 0.000 \\
& $80-200-5$ & 0.000 & 0.000 & 0.000 \\
$120-10-0.625 / 0.5$ & $120-20-0.625 / 0.5$ & 0.760 & 0.781 & 0.166 \\
& $120-200-0.625 / 0.5$ & 0.000 & 0.000 & 0.000 \\
$120-10-1.25 / 1$ & $120-20-1.25 / 1$ & 0.998 & 0.012 & 0.997 \\
& $120-200-1.25 / 1$ & 0.000 & 0.000 & 0.000 \\
$120-10-2.5 / 2$ & $120-20-2.5 / 2$ & 0.060 & 0.002 & 0.109 \\
& $120-200-2.5 / 2$ & 0.000 & 0.000 & 0.000 \\
$120-10-5$ & $120-20-5$ & 0.000 & 0.000 & 0.017 \\
& $120-200-5$ & 0.000 & 0.000 & 0.000 \\
\hline
\end{tabular}

Table 8. Dissimilarities ( $p$ values, student $t$-tests) between the images using the same $\mathrm{kVp}$ and $\mathrm{mAs}$ with varying section thickness in each CT scanner.

\begin{tabular}{ccccc}
\hline \multirow{2}{*}{ (I) Image Code } & (J) Image Code & \multicolumn{3}{c}{ Sig. $(p$ Values, Student $t$-Tests) } \\
\cline { 3 - 5 } & & 16-MDCT & 64-MDCT & 80-MDCT \\
\hline $80-10-0.625 / 0.5$ & $80-10-1.25 / 1$ & 0.004 & 0.348 & 0.029 \\
& $80-10-5$ & 0.888 & 0.012 & 0.013 \\
$80-20-0.625 / 0.5$ & $80-20-1.25 / 1$ & 0.480 & 0.878 & 0.246 \\
& $80-20-5$ & 0.000 & 0.007 & 0.000 \\
$80-50-0.625 / 0.5$ & $80-50-1.25 / 1$ & 1.000 & 0.029 & 0.228 \\
& $80-50-5$ & 0.000 & 0.000 & 0.000 \\
$80-100-0.625 / 0.5$ & $80-100-1.25 / 1$ & 0.193 & 0.138 & 0.000 \\
& $80-100-5$ & 0.000 & 0.000 & 0.000 \\
$80-200-0.625 / 0.5$ & $80-200-1.25 / 1$ & 0.065 & 0.020 & 0.384 \\
& $80-200-5$ & 0.000 & 0.000 & 0.000 \\
$120-10-0.625 / 0.5$ & $120-10-1.25 / 1$ & 0.108 & 0.229 & 0.000 \\
& $120-10-5$ & 0.075 & 0.000 & 0.001 \\
$120-20-0.625 / 0.5$ & $120-20-1.25 / 1$ & 0.773 & 0.003 & 0.434 \\
& $120-20-5$ & 0.000 & 0.000 & 0.000 \\
$120-50-0.625 / 0.5$ & $120-50-1.25 / 1$ & 0.291 & 0.000 & 0.633 \\
& $120-50-5$ & 0.000 & 0.000 & 0.000 \\
$120-100-0.625 / 0.5$ & $120-100-1.25 / 1$ & 0.003 & 0.000 & 0.980 \\
& $120-100-5$ & 0.000 & 0.000 & 0.000 \\
$120-200-0.625 / 0.5$ & $120-200-1.25 / 1$ & 0.067 & 0.000 & 0.139 \\
& $120-200-5$ & 0.000 & 0.000 & 0.000 \\
\hline
\end{tabular}

Considering the 80-MDCT, inconsequential distinctions were present between the images of the 0.5 - and 1-mm thicknesses at 20,50 and $200 \mathrm{mAs}$ and $\mathrm{kVp}$ of 80 . In addition, there were inconsequential peaks between images of the $0.5-$ and 2-mm thicknesses when considered at $50 \mathrm{mAs}$ and $80 \mathrm{kVp}$, as well as insignificant distinctions between images of the $0.5-$ and 1-mm section thicknesses at 20,50, 100 and $200 \mathrm{mAs}$ and $120 \mathrm{kVp}$. Inconsequential peaks were also seen between images of the 0.5- and 2-mm thicknesses at $120 \mathrm{kVp}$ and $10 \mathrm{mAs}$ (Table 8). 


\subsection{Correlation between Scanners Based on CNR Values}

With respect to the CNR values, the 64-MDCT worked as well as the other CT scanners, although the 16-MDCT produced higher CNR values than the 64-MDCT at $80 \mathrm{kVp}$ and $10 \mathrm{mAs}$ with a $1.25-\mathrm{mm}$ section thickness. The 16-MDCT generally worked better than the 80-MDCT, although the 80-MDCT (Figure 2) produced better CNR values than the 16-MDCT at $80 \mathrm{kVp}$ and $10 \mathrm{mAs}$ with a 2.5-mm section thickness, at $80 \mathrm{kVp}$ and $20 \mathrm{mAs}$ with various cut thicknesses and at $80 \mathrm{kVp}$ and $50 \mathrm{mAs}$ with a 2.5-mm section thickness. Large variations were found between the 16-MDCT and 64-MDCT scanners, having certain exceptions: at $10 \mathrm{mAs}$ and $80 \mathrm{kVp}$ with $0.625-$ or $1.25-\mathrm{mm}$ section thicknesses and at $50 \mathrm{mAs}$ and $120 \mathrm{kVp}$ with a $0.625-\mathrm{mm}$ section thickness (Table 9). Basic differences were also seen in the CNR values between the 16-MDCT and the 80-MDCT scanners at low introduction factors: at $10 \mathrm{mAs}$ and $80 \mathrm{kVp}$ with 2.5- or 5-mm section thicknesses, at $20 \mathrm{mAs}$ and $80 \mathrm{kVp}$ with various section thickness; and at $50 \mathrm{mAs}$ and $80 \mathrm{kVp}$ with $0.625-\mathrm{mm} / 0.5-\mathrm{mm}$ and $1.25-\mathrm{mm} / 1-\mathrm{mm}$ section thicknesses. Change in CNR values at $10 \mathrm{mAs}$ among 1-, 2- and 5-mm slice thickness images can be seen in Figure 3. The distinction in CNR values between the 64-MDCT and 80-MDCT scanners were continually basic. The 64-MDCT not only produced higher CNR values than the other scanners, it furthermore provided superior linearity in the CNR values, which increased with elevated $\mathrm{mAs}, \mathrm{kVp}$ and section thickness.

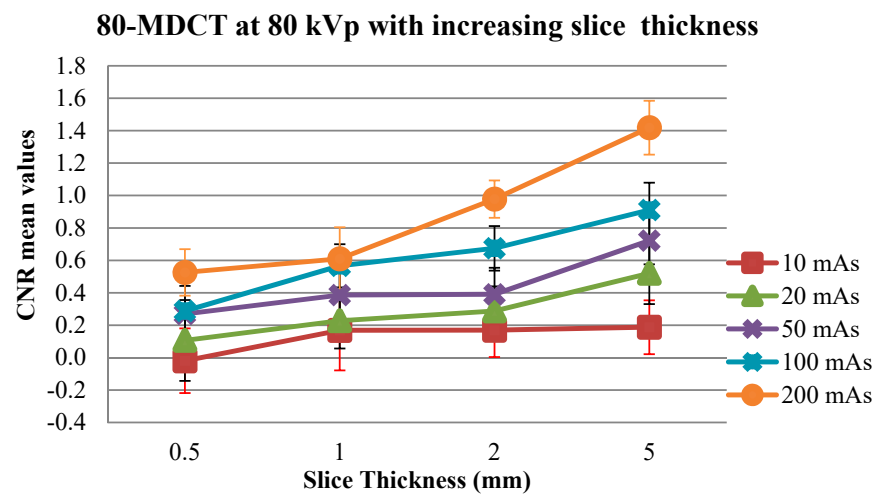

Figure 3. Thicker slice images resulted in higher CNR values at $80 \mathrm{kVp}$ with different $\mathrm{mAs}$ levels for the 80-MDCT scanner. (Note the change in CNR values at $10 \mathrm{mAs}$ among 1-, 2- and 5-mm slice thickness images and the change in CNR values at $50 \mathrm{mAs}$ between 1- and 2-mm slice thickness images.)

Table 9. Dissimilarities ( $p$ values, student $t$-tests) between images of the same factors and section thicknesses utilising various CT scanners.

\begin{tabular}{|c|c|c|c|c|c|}
\hline \multirow{2}{*}{$\mathbf{k V p}$} & \multirow{2}{*}{ mAs } & \multirow{2}{*}{ ST } & \multicolumn{3}{|c|}{ Sig. ( $p$ Values, Student $t$-Tests) } \\
\hline & & & 16-MDCT × 64-MDCT & 16-MDCT $\times 80-\mathrm{MDCT}$ & 64-MDCT $\times 80-\mathrm{MDCT}$ \\
\hline 80 & 10 & $0.625 / 0.5$ & 0.3961 & $<0.001$ & 0.0009 \\
\hline 80 & 10 & 5 & 0.0000 & 0.4788 & 0.0000 \\
\hline 80 & 20 & $0.625 / 0.5$ & 0.0003 & 0.3145 & 0.0009 \\
\hline 80 & 20 & 5 & 0.0000 & 0.3921 & 0.0000 \\
\hline 80 & 50 & $0.625 / 0.5$ & 0.0153 & 0.3027 & 0.0015 \\
\hline 80 & 50 & 5 & 0.0451 & $<0.001$ & 0.0000 \\
\hline 80 & 100 & $0.625 / 0.5$ & 0.0050 & 0.0000 & 0.0000 \\
\hline 80 & 100 & 5 & 0.0000 & 0.0000 & 0.0000 \\
\hline 80 & 200 & $0.625 / 0.5$ & 0.0004 & 0.0009 & 0.0000 \\
\hline 80 & 200 & 5 & 0.0000 & 0.0000 & 0.0000 \\
\hline 120 & 10 & $0.625 / 0.5$ & 0.0276 & 0.0035 & 0.0000 \\
\hline 120 & 10 & 5 & 0.0000 & 0.0018 & 0.0000 \\
\hline 120 & 20 & $0.625 / 0.5$ & 0.0395 & 0.0051 & 0.0000 \\
\hline 120 & 20 & 5 & 0.0000 & 0.0000 & 0.0000 \\
\hline 120 & 50 & $0.625 / 0.5$ & 0.0635 & 0.0002 & 0.0000 \\
\hline 120 & 50 & 5 & 0.0000 & 0.0000 & 0.0000 \\
\hline 120 & 100 & $0.625 / 0.5$ & 0.0002 & 0.0003 & 0.0000 \\
\hline 120 & 100 & 5 & 0.0000 & 0.0000 & 0.0000 \\
\hline 120 & 200 & $0.625 / 0.5$ & 0.0000 & 0.0000 & 0.0000 \\
\hline 120 & 200 & 5 & 0.0000 & 0.0000 & 0.0000 \\
\hline
\end{tabular}




\section{Discussion}

The effect of object size on CNR values was examined from various angles, in terms of scanner type, reconstruction calculation, contrast level of the object and $\mathrm{mAs}$. The outcomes of the modifying factors-mAs, $\mathrm{kVp}$ and section thickness-on the values of CNR were also investigated, and the operation of the various scanners, based on the CNR values, were compared and correlated.

It was seen that the CNR values were considerably affected by adjusting the image reconstruction calculations. Although it was seen that the CNR values were high in the upper level in the soft-tissue reconstruction images in the 16-MDCT and 64-MDCT scanners, some CNR values were found to be higher in the standard reconstruction images than in the other types of algorithmic reconstruction images in the 80-MDCT scanner. This accords with the findings of several different studies [22,23]. As found by Kalender and Khadivi [1], changing the algorithmic reconstruction produces different trademark pixel sound estimates, which are characterised by the reconstruction. For example, a normal pixel sound for soft tissue is $62.1 \mathrm{HU}$, while, for standard and high-goal parts, they are 31.5 and $57.5 \mathrm{HU}$, respectively, for a 1- and 32-mm section thickness. The sound level of CT images is a significant factor, compared to the location of LCD objects $[1,24]$. The outcomes do not show any noteworthy changes in the CNR values among other object estimates, down to the smallest measurable object $(5 \mathrm{~mm})$. It was observed that the ability to identify objects depends on the differentiation standard of the object, as well as its size [19-26]. Step-by-step instructions to control undefined, minor lesions (e.g., nodules in CT lung screening) have become a significant concern. Although most very small nodules are benign, a few will end up being cancer-causing [27]. Some minor lesions, (e.g., small-cell carcinomas) grow in rapid increments, with a mean volume multiplying time of around 149 days [28]. Consequently, early intervention is vital to implement treatment [27]. The exact measurement and precise identification of the size of pulmonary nodules and various injuries are necessary in various clinical cases. This allows monitoring of the impacts of chemotherapy and injury development at follow-up, which may reveal risk [29]. An appraisal method based on estimated CNR values is not good enough to determine the consequences of object size on the capacity of the LCD.

Overall, a higher $\mathrm{kV}$ p produced superior CNR values in all the $\mathrm{CT}$ scanners. Specifically, there were critical elevations in CNR values when the voltage was elevated from 80 to $120 \mathrm{kVp}$. The impacts of $\mathrm{kVp}$ on the quality of image relative to the $\mathrm{CNR}$ values were as anticipated, with elevating $\mathrm{kVp}$ increasing photon entrance and the radiation portion when the other factors were notably fixed, even though the radiation portion is not straight with $\mathrm{kVp}$. Subsequently, the noise is diminished and the CNR is increased $[9,30]$.

Higher mAs levels mainly produced better CNR values, especially for thicker sections. As expected, the CNR is improved with increasing mAs because this decreases the image noise. The radiation portion is directly related to the mAs [31,32]. With thicker sections, the CNR values mostly got elevated. The impact of section thickness on the imaging ability of the CT scanners was anticipated because thicker sections decrease image noise so the image quality improves [33]. As indicated above, there were inconsequential differences in the CNR values for the $0.625-\mathrm{mm} / 0.5-\mathrm{mm}$ and $1.25-\mathrm{mm} / 1-\mathrm{mm}$ section thicknesses at all $\mathrm{mAs}$ levels, as well as between the $0.625-\mathrm{mm} / 0.5-\mathrm{mm}$ and $2.5-\mathrm{mm} / 2-\mathrm{mm}$ section thicknesses, especially at lower introduction factors. The noise elevates with thinner slices if the radiation dose is not allowed to escalate $[9,30,33]$. In any case, thinner sections give high-goal, isotropic image informational indices, and subsequently the through-plane, incomplete-volume averaging impacts are limited and the image is upgraded $[1,34,35]$.

With respect to the CNR value estimations, the 64-MDCT scanner worked the best, whilst the 16-MDCT scanner worked better than the 80-MDCT scanner, for the most part. There were important and consequential differences between the 16-MDCT and 64-MDCT scanners. The specific properties and framework specifications of each scanner determined their imaging capabilities. The CT scanner manufacturer, model, geometry, tube details and locator configuration all determine the noise and image clarity, which all influence the imaging and image quality $[9,26,36]$. This study was restricted by 
using only one scanner arrangement, from one manufacturer, and by the smallest quantifiable object being $5 \mathrm{~mm}$ in diameter.

\section{Conclusions}

The LCD assessment technique, based on the CNR values, was sensitive to the quantification of the impacts of $\mathrm{kVp}$, mAs and section thickness on image quality. This technique was also successful in assessing the impacts on image quality of various reconstruction calculations and objects at different levels, based on the CNR values. However, it should be noted that the smallest object size that could be estimated, using this technique with the Catphan ${ }^{\circledR} 600$, was $5 \mathrm{~mm}$. Smaller objects could not be distinguished with any precision without estimating the outside of the object in order to determine the object's mean CT value. In that respect, no notable CNR changes were found between various object sizes. Thus, LCD perceptibility is not only dictated by object differentiation, but also by object size. Therefore, this method is not an appropriate method to measure LCD detectability performance. It is also not possible for this phantom design to appraise an object of the same size and contrast at different position levels inside the phantom. It is a time consuming, inconvenient and vexatious as it necessitates scrutiny of an immensely vast quantity of data. Furthermore, authenticity of this technique is comparatively low as human eyewitnesses were not incorporated in the procedure. Thus, utilisation of Catphan ${ }^{\circledR} 600$ should not be considered as an appurtenant apparatus for image optimisation intentions and regularly based appraisals. In addition, volume CT dose index $\left(\mathrm{CTDI}_{\mathrm{vol}}\right)$, which is a systematised calculation of the radiation output coming out of a CT system, that permits users to find out the quantity of radiation emitted and equates the radiation output between various scanners, can be considered in future research. Another point to be mentioned is that the dose-length product (DLP), which is directly connected to the patient (stochastic) dangers, can also be utilised for future similar research.

Author Contributions: Conceptualisation, H.M.A. and H.A.A.; Data curation, H.M.A. and H.A.A.; Formal analysis, H.M.A. and H.A.A.; Funding acquisition, H.A.A.; Investigation, H.M.A.; Methodology, H.M.A. and H.A.A.; Project administration, H.M.A. and H.A.A.; Resources, H.M.A. and H.A.A.; Software, H.M.A.; Supervision, H.M.A.; Validation, H.M.A. and H.A.A.; Visualisation, H.M.A. and H.A.A.; Writing-original draft, H.M.A. and H.A.A.; and Writing - review and editing, H.A.A. All authors have read and agreed to the published version of the manuscript.

Funding: This research received no external funding.

Conflicts of Interest: The authors have no known conflicts of interest to declare.

\section{References}

1. Kalender, W.; Khadivi, K. Computed Tomography: Fundamentals, System Technology, Image Quality, Applications, 3rd ed.; Medical Physics, Publicis Publishing: Erlangen, Germany, 2011.

2. Berrington de Gonzalez, A.; Mahadevappa, M.; Kwang-Pyo, K.; Mythreyi, B.; Mythreyi, R.; Mettler, F.; Land, C. Projected cancer risks from computed tomographic scans performed in the United States in 2007. Arch. Intern. Med. 2009, 169, 2071-2077. [CrossRef]

3. Martinsen, A.; Saether, H.; Olsen, D.; Wolff, P.; Skaane, P. Improved image quality of low-dose thoracic CT examinations with a new postprocessing software. J. Appl. Clin. Med. Phys. 2010, 11, 132. [CrossRef]

4. Edyvean, S.; Lewis, M.; Britten, A. Radiation Dose Metrics and the Effect of CT Scan Protocol Parameters. In Radiation Dose from Multidetector CT; Medical Radiology; Tack, D., Kalra, M., Gevenois, P., Eds.; Springer: Berlin/Heidelberg, Germany, 2012.

5. Liu, J.; Hu, Y.; Yang, J.; Chen, Y.; Shu, H.; Luo, L.; Feng, Q.; Gui, Z.; Coatrieux, G. 3D Feature Constrained Reconstruction for Low-Dose CT Imaging. IEEE Trans. Circuits Syst. Video Technol. 2018, 28, 1232-1247. [CrossRef]

6. Liu, J.; Ma, J.; Zhang, Y.; Chen, Y.; Yang, J.; Shu, H.; Luo, L.; Coatrieux, G.; Yang, W.; Feng, Q.; et al. Discriminative Feature Representation to Improve Projection Data Inconsistency for Low-Dose CT Imaging. IEEE Trans. Med. Imaging 2017, 36, 2499-2509. [CrossRef] 
7. Chen, Y.; Shi, L.; Feng, Q.; Yang, J.; Shu, H.; Luo, L.; Coatrieux, J.L.; Chen, W. Artifact Suppressed Dictionary Learning for Low-Dose CT Image Processing. IEEE Trans. Med. Imaging 2014, 33, 227-2292. [CrossRef]

8. Hara, A.K.; Paden, R.G.; Silva, A.C.; Kujak, J.L.; Lawder, H.J.; Pavlicek, W. Iterative reconstruction technique for reducing body radiation dose in CT: Feasibility study. Am. J. Roentgenol. 2009, 193, 764-771. [CrossRef]

9. Alsleem, H.; Davidson, R. Factors affecting contrast-detail performance in computed tomography: A review. J. Med. Imaging Radiat. Sci. 2013, 44, 62-70. [CrossRef] [PubMed]

10. Hernandez-Giron, I.; Geleijns, J.; Calzado, A.; Veldkamp, W. Automated assessment of low contrast sensitivity for CT systems using a model observer. Med. Phys. 2011, 38, S25-S35. [CrossRef]

11. Pascoal, A.; Lawinski, C.; Honey, I.; Blake, P. Evaluation of a software package for automated quality assessment of contrast detail images-comparison with subjective visual assessment. Phys. Med. Biol. 2005, 50, 5743-5757. [CrossRef]

12. Oda, S.; Utsunomiya, D.; Funama, Y.; Katahira, K.; Honda, K.; Tokuyasu, S.; Vembar, M.; Yuki, H.; Noda, K.; Oshima, S.; et al. A knowledge-based iterative model reconstruction algorithm: Can super-low-dose cardiac CT be applicable in clinical settings? Acad. Radiol. 2014, 21, 104-110. [CrossRef] [PubMed]

13. Nakaura, T.; Kidoh, M.; Sakaino, N.; Utsunomiya, D.; Oda, S.; Kawahara, T.; Harada, K.; Yamashita, Y. Low-contrast and low-radiation dose protocol for cardiac CT of thin adults in 256-row CT: Usefulness of low tube-voltage scans and the hybrid iterative reconstruction algorithm. Int. J. Cardiovasc. Imaging 2013, 29, 913-923. [CrossRef]

14. Mehta, D.; Thompson, R.; Morton, T.; Dhanantwari, A.; Shefer, E. Iterative model reconstruction: Simultaneously lowered computed tomography radiation dose and improved image quality. Med. Phys. Int. J. 2013, 1, 147-155.

15. Li, T.; Zhang, Y.; Wang, Y.; Gao, J.; Jiang, Y. Chest CT with iterative reconstruction algorithms for airway stent evaluation in patients with malignant obstructive tracheobronchial diseases. Medicine (Baltimore) 2016, 95, e4873. [CrossRef] [PubMed]

16. Zhang, F.; Yang, L.; Song, X.; Li, Y.N.; Jiang, Y.; Zhang, X.H.; Ju, H.Y.; Wu, J.; Chang, R.P. Feasibility study of low tube voltage $(80 \mathrm{kVp})$ coronary $\mathrm{CT}$ angiography combined with contrast medium reduction using iterative model reconstruction (IMR) on standard BMI patients. Br. J. Radiol. 2016, 89, 20150766. [CrossRef] [PubMed]

17. Khawaja, R.D.A.; Singh, S.; Blake, M.; Harisinghani, M.; Choy, G.; Karosmangulu, A.; Padole, A.; Do, S.; Brown, K.; Thompson, R.; et al. Ultra-low-dose abdominal MDCT: Using a knowledge-based Iterative Model Reconstruction technique for substantial dose reduction in a prospective clinical study. Eur. J. Radiol. 2015, 84, 2-10. [CrossRef] [PubMed]

18. Xin, X.; Shen, J.; Yang, S.; Liu, S.; Hu, A.; Zhu, B.; Jiang, Y.; Li, B.; Zhang, B. Improved image quality of low-dose $\mathrm{CT}$ combined with an iterative model reconstruction algorithm for response assessment in patients after treatment of malignant tumor. Quant. Imaging Med. Surg. 2018, 8, 648-657. [CrossRef] [PubMed]

19. Baker, M.; Dong, F.; Primak, A.; Obuchowski, N.; Einstein, D.; Gandhi, N.; Herts, B.; Purysko, A.; Remer, E.; Vachani, N. Contrast-to-Noise Ratio and Low-Contrast Object Resolution on Full-and Low-Dose MDCT: SAFIRE Versus Filtered Back Projection in a Low-Contrast Object Phantom and in the Liver. Am. J. Roentgenol. 2012, 199, 8-18. [CrossRef]

20. Heyer, C.; Mohr, P.; Lemburg, S.; Peters, S.; Nicolas, V. Image Quality and Radiation Exposure of Pulmonary CT Angiography with a 100- or 120-kVp Protocol: Prospective Randomized Study 1. Radiology 2007, 245, 577-583. [CrossRef]

21. Pallant, J. SPSS Survival Manual: A Step-by-step Guide to Data Analysis using IBM SPSS; McGraw-Hill: Maidenhead, UK, 2013.

22. Feng, C.; Zhu, D.; Zou, X.; Li, A.; Hu, X.; Li, Z.; Hu, D. The combination of a reduction in contrast agent dose with low tube voltage and an adaptive statistical iterative reconstruction algorithm in CT enterography. Medicine 2018, 97, e0151. [CrossRef]

23. Keller, M.R.; Kessler, R.M.; Brooks, R.A.; Kirkland, L.R. Optimum energy for performing CT iodinated contrast studies. Br. J. Radiol. 1980, 53, 576-579. [CrossRef]

24. Bache, S.T.; Stauduhar, P.J.; Liu, X.; Loyer, E.M.; John, R.X. Quantification of clinical feedback on image quality differences between two CT scanner models. J. Appl. Clin. Med. Phys. 2017, 18, 163-169. [CrossRef]

25. Davidson, R. Radiographic Contrast-Enhancement Masks in Digital Radiography. Ph.D. Thesis, University of Sydney, Sydney, Australia, 2007. 
26. Faulkner, K.; Moores, B. Noise and contrast detection in computed tomography images. Phys. Med. Biol. 1984, 29, 329-339. [CrossRef] [PubMed]

27. MacMahon, H.; Austin, J.; Gamsu, G.; Herold, C.; Jett, J.; Naidich, D.; Patz, E.; Swensen, S. Guidelines for management of small pulmonary nodules detected on CT scans: A statement from the Fleischner Society. Radiology 2005, 237, 395-400. [CrossRef]

28. Hasegawa, M.; Sone, S.; Takashima, S.; Li, F.; Yang, Z.; Maruyama, Y.; Watanabe, T. Growth rate of small lung cancers detected on mass CT screening. Br. J. Radiol. 2000, 73, 1252-1259. [CrossRef]

29. Wormanns, D.; Diederich, S.; Lentschig, M.; Winter, F.; Heindel, W. Spiral CT of pulmonary nodules: Interobserver variation in assessment of lesion size. Eur. Radiol. 2000, 10, 710-713. [CrossRef]

30. Seibert, J. Tradeoffs between image quality and dose. Pediatr. Radiol. 2004, 34, 183-195. [CrossRef]

31. Funama, Y.; Awai, K.; Nakayama, Y.; Kakei, K.; Nagasue, N.; Shimamura, M.; Sato, N.; Sultana, S.; Morishita, S.; Yamashita, Y. Radiation Dose Reduction without Degradation of Low-Contrast Detectability in Abdominal Multisection CT with a Low-Tube-Voltage Technique: Phantom Study 1. Radiology 2005, 237, 905-910. [CrossRef]

32. Toth, T. Image Quality in CT: Challenges and Perspectives. In Radiation Dose from Multidetector CT, 2nd ed.; Tack, D., Kalra, M.K., Gevenois, P.A., Eds.; Springer: Berlin/Heidelberg, Germany, 2012; pp. 81-100.

33. Von Falck, C.; Galanski, M.; Shin, H. Informatics in Radiology: Sliding-Thin-Slab Averaging for Improved Depiction of Low-Contrast Lesions with Radiation Dose Savings in Thin-Section CT. Radiographics 2010, 30, 317-326. [CrossRef]

34. Rubin, G. 3-D imaging with MDCT. Eur. J. Radiol. 2003, 45, S37-S41. [CrossRef]

35. Hsieh, J. Computed Tomography: Principles, Design, Artifacts, and Recent Advances, 2nd ed.; SPIE: Bellingham, DC, USA, 2009.

36. Mahesh, M. MDCT Physics: The Basics-Technology, Image Quality and Radiation Dose, 1st ed.; Lippincott Williams \& Wilkins: Philadelphia, PA, USA, 2009.

(C) 2020 by the authors. Licensee MDPI, Basel, Switzerland. This article is an open access article distributed under the terms and conditions of the Creative Commons Attribution (CC BY) license (http://creativecommons.org/licenses/by/4.0/). 